\title{
ARTICLE \\ Osimertinib successfully combats EGFR-negative glioblastoma cells by inhibiting the MAPK pathway
}

\author{
Cheng Chen ${ }^{1,2}$, Chuan-dong Cheng ${ }^{1,2,3,4}$, Hong Wu ${ }^{1,5}$, Zuo-wei Wang ${ }^{1,2}$, Li Wang ${ }^{1}$, Zong-ru Jiang ${ }^{1,5}$, Ao-li Wang ${ }^{1,5}$, Chen Hu $\mathrm{Hu}^{1,5}$, \\ Yong-fei Dong ${ }^{3,4}$, Wan-xiang Niu ${ }^{3,4}$, Shuang Qi ${ }^{1,5}$, Zi-ping Qi ${ }^{1,5}$, Jing Liu ${ }^{1,5}$, Wen-chao Wang ${ }^{1,5}$, Chao-shi Niu ${ }^{3,4}$ and Qing-song Liu ${ }^{1,2,5,6,7}$
}

\begin{abstract}
Glioblastoma (GBM) patients have extremely poor prognoses, and currently no effective treatment available including surgery, radiation, and chemotherapy. MAPK-interacting kinases (MNK1/2) as the downstream of the MAPK-signaling pathway regulate protein synthesis in normal and tumor cells. Research has shown that targeting MNKs may be an effective strategy to treat GBM. In this study we investigated the antitumor activity of osimertinib, an FDA-approved epidermal growth factor receptor (EGFR) inhibitor, against patient-derived primary GBM cells. Using high-throughput screening approach, we screened the entire panel of FDA-approved drugs against primary cancer cells derived from glioblastoma patients, found that osimertinib $(3 \mu \mathrm{M})$ suppressed the proliferation of a subset (10/22) of EGFR-negative GBM cells ( $>50 \%$ growth inhibition). We detected the gene expression difference between osimertinib-sensitive and -resistant cells, found that osimertinib-sensitive GBM cells displayed activated MAPK-signaling pathway. We further showed that osimertinib potently inhibited the MNK kinase activities with $\mathrm{IC}_{50}$ values of $324 \mathrm{nM}$ and $48.6 \mathrm{nM}$, respectively, against MNK1 and MNK2 kinases; osimertinib (0.3-3 $\mu \mathrm{M})$ dose-dependently suppressed the phosphorylation of eukaryotic translation initiation factor 4E (elF4E). In GBM patient-derived xenografts mice, oral administration of osimertinib (40 mg. $\mathrm{kg}^{-1} \cdot \mathrm{d}^{-1}$, for 18 days) significantly suppressed the tumor growth (TGI $=74.5 \%$ ) and inhibited elF4E phosphorylation in tumor cells. Given the fact that osimertinib could cross the blood-brain barrier and its toxicity was well tolerated in patients, our results suggest that osimertinib could be a new and effective drug candidate for the EGFR-negative GBM patients.
\end{abstract}

Keywords: glioblastoma; osimertinib; temozolamide; MAPK-signaling pathway; MNK; eukaryotic translation initiation factor 4E

Acta Pharmacologica Sinica (2021) 42:108-114; https://doi.org/10.1038/s41401-020-0418-2

\section{INTRODUCTION}

Glioblastoma (GBM) is among the most aggressive and common primary brain tumors in adults. GBM patients have extremely poor prognoses, and currently no treatment (including surgery, radiation, and chemotherapy) is able to extend the median survival beyond fifteen months [1, 2]. Despite accumulating knowledge of GBM pathology, little progress has been made in drug discovery. Genetic heterogeneity, the blood-brain barrier (BBB), and unknown genetic abnormalities in GBM have limited the effectiveness of therapeutic approaches and anticancer agents [3]. Therefore, there is an urgent need for the development of more effective therapies.

Protein synthesis is a fundamental cellular process indispensable for both normal cellular function and oncogenesis [4]. MNK1 and MNK2 are downstream of the MAPK-signaling pathway and regulate protein synthesis through binding to eukaryotic translation initiation factor $4 \mathrm{G}$ (elF4G) and phosphorylating eukaryotic translation initiation factor 4E (elF4E) $[5,6]$. elF4E-mediated mRNA translation plays an important role in oncogenic transformation, and an elevated level of elF4E expression/activity has been observed in many tumors [7-9]. Recent studies demonstrated that pharmacological inhibition of MNKs could block elF4E-mediated translation and thus suppress GBM cell proliferation and slowdown tumor growth in vivo $[4,10]$. These findings suggest that targeting MNKs may be an effective strategy to treat GBM. Here, we report that the EGFR inhibitor osimertinib could induce cell death in a panel of EGFR-negative patient-derived primary GBM cells by targeting MNKs. Our findings provide evidence to expand the application of osimertinib to GBM patients.

\section{MATERIALS AND METHODS}

Patients characteristics

From January 2017 to March 2018, 31 GBM patient samples were collected from the First Affiliated Hospital of the University of Science and Technology of China (Hefei, China),

\footnotetext{
${ }^{1}$ High Magnetic Field Laboratory, Key Laboratory of High Magnetic Field and Ion Beam Physical Biology, Hefei Institutes of Physical Science, Chinese Academy of Sciences, Hefei 230031, China; ${ }^{2}$ University of Science and Technology of China, Hefei 230036, China; ${ }^{3}$ Department of Neurosurgery, The First Affiliated Hospital of USTC, Division of Life Sciences and Medicine, University of Science and Technology of China, Hefei 230036, China; ${ }^{4}$ Anhui Province Key Laboratory of Brain Function and Brain Disease, Hefei 230031 , China; ${ }^{5}$ Precision Medicine Research Laboratory of Anhui Province, Hefei 230088, China; ${ }^{6}$ Precision Targeted Therapy Discovery Center, Institute of Technology Innovation, Hefei Institutes of Physical Science, Chinese Academy of Sciences, Hefei 230088, China and 'Institutes of Physical Science and Information Technology, Anhui University, Hefei 230601, China

Correspondence: Wen-chao Wang (wwcbox@hmfl.ac.cn) or Chao-shi Niu (niuchaoshi@ustc.edu.cn) or Qing-song Liu (qsliu97@hmfl.ac.cn)

These authors contributed equally: Cheng Chen, Chuan-dong Cheng, Hong Wu, Zuo-wei Wang
}

Received: 6 January 2020 Accepted: 12 April 2020

Published online: 12 May 2020 
and 22 patient-derived cancer cells (PDCs) were established for subsequent experiments. The median age at diagnosis was 48.6 years (6-71), and the male:female ratio was 10:12. All studies performed with human specimens were done with approval from the First Affiliated Hospital of the University of Science and Technology of China (Hefei, China). Ethical approval and informed consent were obtained for the use of human samples.

Culture of primary human GBM cells

The tumor tissue samples were obtained from newly and recurrent GBM patients. Primary cells were collected by centrifugal filtration and plated in DMEM/F-12 medium (Corning, NY, USA) with Gibco B-27 Serum Free Supplement (Sigma-Aldrich, MO, USA), bFGF (40 ng/mL) (Sigma-Aldrich, MO, USA), EGF $(20 \mathrm{ng} / \mathrm{mL})$ (Sigma-Aldrich, MO, USA), and $1 \%$ insulin (Sigma-Aldrich, MO, USA). The cells were cultured at $37^{\circ} \mathrm{C}$ in a humidified $5 \% \mathrm{CO}_{2}$ incubator, and the medium was changed every 2 days. We passaged cells using $0.05 \%$ pancreatin (Corning, NY, USA) when PDCs reached $80 \%$ confluence.

High-throughput drug sensitivity analysis

We used the high-throughput drug sensitivity analysis to evaluate the effect of different clinical drugs on PDC cells. The primary cells grown in DMEM/F-12 medium (Corning, NY, USA) supplemented with B-27 (Sigma-Aldrich, MO, USA) were seeded in a 384-well plate (Corning, NY, USA) at a density of 2000 cells per well. The clinical drugs (MedChem Express, Shanghai, China) at multiple concentrations $(3,1$, and $0.3 \mu \mathrm{M})$ were added into the plates using an automated liquid handling system (PerkinElmer, MA, USA) after the primary cells were cultured for $12 \mathrm{~h}$. Cell viability was analyzed using CellTiter-Glo ${ }^{\mathrm{TM}}$ kits (Promega, WI, USA) after $72 \mathrm{~h}$ of drug treatment. After normalization, the inhibition rate of each drug was calculated using the following formula:

$$
\begin{aligned}
\text { Inhibition rate }(\%)= & 100 \%-\left(\mathrm{RLU}_{\text {Drug }}-\mathrm{RLU}_{\text {Background }}\right) / \\
& \left(\mathrm{RLU}_{\text {DMSO }}-\mathrm{RLU}_{\text {Background }}\right) \times 100 \%
\end{aligned}
$$

\section{RNA-sequencing analysis}

Total RNA was prepared using the RNeasy Miniprep Kit (Qiagen, Hilden, Germany) and checked for quality using an Agilent Bioanalyzer with an RNA integrity number (RIN) for all primary GBM cells. The data were profiled with human RNA-sequencing analysis (SmartQuerier Biomedicine, Shanghai, China). Differential gene expression analysis was performed using the DESeq2 R package (1.23.0).

\section{Signal-transduction pathway analysis}

The primary human cancer cells were treated with DMSO, osimertinib, tomivosertib, and erlotinib for $2 \mathrm{~h}$ before immunoblotting. The cells were then collected and lysed in RIPA buffer (Beyotime, Shanghai, China) with a protease inhibitor cocktail (Sigma-Aldrich, MO, USA). The following antibodies were used at a range of antibody concentrations as indicated by the manufacturers to probe for specific proteins: EGFR (\#4267), phospho-ERK (\#4370), ERK (\#4695), phospho-elF4E (\#9741), elF4E (\#2067), elF4G (\#2498), elF4A (\#2013), GAPDH (\#5174), and a-Tubulin (\#2144) were all from Cell Signaling Technology (Danvers, MA, USA).

\section{Biochemical kinase assay}

The fluorescence resonance energy-transfer-based Z'-LYTE kinase assay kit (Invitrogen, CA, USA) was used to evaluate the $I_{50}$ value of osimertinib for inhibition of MNK1 and MNK2 kinases. The reaction was performed on a 384-well plate with a $10 \mu \mathrm{L}$ reaction volume per well containing $2 \mu \mathrm{M}$ peptide substrate in a reaction buffer and $2.5 \mu \mathrm{L}$ MNK1 or MNK2 protein (20 ng) with a serial 3fold dilution of osimertinib $(2.5 \mu \mathrm{L}, 10 \mu \mathrm{M}-1.5 \mathrm{nM})$. The ATP concentration was optimized, $10 \mu \mathrm{M}$ ATP for MNK1 and MNK2 kinase. After 1-h incubation, a reaction was developed and terminated, and the fluorescence was measured with an automated plate reader (SpectraMax I3, MD, CA, USA). A doseresponse curve was fitted using Prism 5.0 (GraphPad Software Inc., CA, USA).

Proliferation assay

The primary human cancer cells were grown in 96-well culture plates (2500-3000 cells/well). Various concentrations (osimertinib: $10 \mu \mathrm{M}, 8 \mu \mathrm{M}, 6 \mu \mathrm{M}, 4 \mu \mathrm{M}, 1 \mu \mathrm{M}, 0.5 \mu \mathrm{M}, 0.1 \mu \mathrm{M}, 0 \mu \mathrm{M}$; Tomivosertib: $50 \mu \mathrm{M}, 40 \mu \mathrm{M}, 25 \mu \mathrm{M}, 15 \mu \mathrm{M}, 10 \mu \mathrm{M}, 3 \mu \mathrm{M}, 1 \mu \mathrm{M}, 0 \mu \mathrm{M}$; Erlotinib: $20 \mu \mathrm{M}, 15 \mu \mathrm{M}, 10 \mu \mathrm{M}, 8 \mu \mathrm{M}, 6 \mu \mathrm{M}, 4 \mu \mathrm{M}, 2 \mu \mathrm{M}, 0 \mu \mathrm{M})$ of compounds were added into the plates after adherent cells were cultured for $12 \mathrm{~h}$. Cell proliferation was determined after treatment with compounds for $72 \mathrm{~h}$. The proliferation assay was measured using the Cell Titer-Glo ${ }^{\mathrm{TM}}$ assay (Promega, WI, USA) according to the manufacturer's instructions. Luminescence was measured by a multilabel reader (Envision, PerkinElmer, MA, USA). Data were normalized to control (DMSO) and $\mathrm{GI}_{50}$ values were calculated using Prism 5.0 (GraphPad Software Inc., CA, USA).

Colony-formation assay

GBM primary cells were trypsinized and dispensed into individual wells of six-well tissue culture dishes at a density of $1 \times 10^{4}$ cells per well. Cells were maintained in a humidified $5 \% \mathrm{CO}_{2}$ incubator at $37^{\circ} \mathrm{C}$ for 15 days, and continuously treated with serially diluted osimertinib, erlotinib, and tomivosertib. On the 15th day, the number of cells in each well was determined by crystal violet staining, and each experiment was repeated three times.

Wash-out experiment

The GBM primary cells were treated with $10 \mu \mathrm{M}$ osimertinib for $4 \mathrm{~h}$ before they were washed with PBS three times. Then, cells were incubated in medium for the indicated time periods $(0,15,30,45$, and $60 \mathrm{~min}$ ) before they were collected and lysed. Anti-elF4E and anti-phospho-elF4E antibodies were used for immunoblotting.

Cap-binding assay

The primary cancer cells were lysed in buffer A $[10 \mathrm{mM}$ Tris-HCL (pH 7.6), $140 \mathrm{mM} \mathrm{KCl}, 4 \mathrm{mM} \mathrm{MgCl} 2,1 \mathrm{mM}$ DTT, $1 \mathrm{mM}$ EDTA, and protease inhibitors, supplemented with 1\% NP-40], and the cell lysates (p0013, Beyotime, Shanghai, China) were incubated overnight at $4{ }^{\circ} \mathrm{C}$ with $50 \mu \mathrm{L}$ of the mRNA cap analog $\mathrm{m}^{7} \mathrm{GTP}$ Sepharose (GE Healthcare, IL, USA) in buffer A under constant and gentle agitation. The protein complex Sepharose beads were washed with buffer A supplemented with $0.5 \% \mathrm{NP}-40$ and the eIF4E-associated complex was resolved by SDS-PAGE and Western blotting. The following antibodies were used according to the manufacturers' instructions: rabbit anti-elF4G, mouse anti-elF4E, rabbit anti-elF4A, and anti-GAPDH [11]. All antibodies were purchased from Cell Signaling Technology (Danvers, MA, USA)

Puromycin incorporation assay

The cells were treated with puromycin $(10 \mu \mathrm{M})$ (MedChem Express, Shanghai, China) for 15 min, lysed, and processed for immunoblotting as previously described [12].

Animal models

Five-week-old female NCG mice were purchased from the Nanjing Biomedical Research Institute of Nanjing University (Nanjing, China). The studies were approved by the Hefei Institutes of Physical Science Ethics Committee, Chinese Academy of Sciences (Hefei, China). All animals were housed in a specific pathogen-free facility and maintained according to the animal care regulations of Hefei Institutes of Physical Science, Chinese Academy of Sciences (Hefei, China). The GBM patient samples were provided by the First Affiliated Hospital of the University of Science and Technology of China (Hefei, China). GBM patient cells $\left(1 \times 10^{5}\right)$ in 
PBS were injected into the right brain of NCG mice. Osimertinib and temozolamide were delivered daily in a $\mathrm{HKI}$ solution $(0.5 \%$ Methocellulose/0.4\% Tween 80 in $\mathrm{dd}_{2} \mathrm{O}$ ) by orally gavage for 18 days. Daily oral administration was initiated after 2 weeks. Body weight were recorded every day. The brains of mice were obtained at the time of euthanasia.

\section{Statistical analysis}

Prism 5.0 (GraphPad Software Inc., San Diego, CA, USA) and R were used for all statistical analyses ( $t$-tests, Kruskal-Wallis tests, Fisher's exact tests). Three independent replicates were performed for each experiment. Data are presented as mean \pm standard deviation (SD), and differences between groups were assessed by a paired $t$-test and accepted as significant at $P<0.05$.

\section{RESULTS}

Osimertinib effectively inhibits primary GBM cell proliferation "Drug repurposing" is one of the most efficient approaches to search for new available therapies for unmet clinical demands. Using a high-throughput screening approach, we screened the entire panel of FDA-approved drugs in 22 primary cancer cells derived from glioblastoma patients (Table 1). The cells were exposed to these drugs at three concentrations $(1,3$, and $10 \mu \mathrm{M})$, and their antiproliferative effects were analyzed with CellTiter-Glo assays. A proliferation inhibition index was determined for each drug by comparing the mean effect of the drug (assessed in triplicates) with that of the vehicle control. From the results, we identified seven agents with potent inhibitory effects against GBM primary cancer cells, one of which was osimertinib, an irreversible epidermal growth factor receptor (EGFR) kinase inhibitor used for metastatic non-smallcell lung cancer patients with the EGFR T790M mutation. Among the 22 primary GBM cell samples tested, osimertinib displayed potent efficacy against 10 samples with greater than $50 \%$ growth inhibition at the $3 \mu \mathrm{M}$ concentration. For the remaining 11 samples, no apparent antiproliferative activity was observed with $3 \mu \mathrm{M}$ osimertinib (Fig. 1a). Interestingly, we noticed that osimertinib was much more potent than gefitinib and erlotinib, which are two well-known EGFR inhibitors (Fig. 1b). To understand the mechanism behind the difference in osimertinib sensitivity between these primary cells, we used RNAsequencing to profile gene expression differences between the sensitive and resistant cells. The genes upregulated in the sensitive cells were enriched in pathways related to MAPKsignaling (Fig. 1C). In addition, KRAS signaling-related genes (including MAPK) were enriched in GSEA (Fig. 1d). These results

\begin{tabular}{|lll|}
\hline \multicolumn{2}{|l|}{ Table 1. $\quad$ Baseline patient characteristics $(n=22)}$. & \\
\hline Variable & Patients $(n=22)$ & $\%$ \\
\hline $\begin{array}{l}\text { Age-year } \\
\quad \text { Median/Range }\end{array}$ & $48.6 /(6-71)$ & \\
Sex & & \\
$\quad$ Male/Female & $10 / 12$ & $45.45 / 54.55$ \\
Location & & 40.91 \\
Right & 9 & 22.73 \\
Middle & 5 & 36.36 \\
Left & 8 & \\
WHO grade & & 9.09 \\
I & 2 & 31.82 \\
II & 7 & 18.18 \\
III & 4 & 40.91 \\
IV & 9 & \\
\hline
\end{tabular}

suggested that osimertinib was more effective in GBM samples with activated MAPK-signaling than in those without activated MAPK-signaling.

Targeting MNKs effectively inhibits EGFR-negative GBM cells MNKs are downstream effectors of MAPK-signaling, and previous studies have already shown that MNK2 is one of the off-targets of osimertinib by kinome profiling assay. To further elucidate the effect of osimertinib on MNKs, we tested osimertinib with the Z'-LYTE kinase biochemical assay, in which osimertinib displayed an $\mathrm{IC}_{50}$ value of $324 \mathrm{nM}$ and $48.6 \mathrm{nM}$ against MNK1 and MNK2, respectively (Fig. 2a). We then modeled a potential configuration of osimertinib docked into the MNK2 X-ray structure (PDB ID: $6 \mathrm{CJH}$ ) and found that osimertinib could fit well in the DFG-in kinase active conformation of MNK2 and adopted the type-I binding mode (Fig. $2 \mathrm{~b}$ left). In addition, in the solid surface conformation, osimertinib adopted a reversible binding mode with MNK2 (Fig. 2b right). The imidazole core formed a hydrogen bond with Met 162 in the hinge binding area, and the methoxy formed a hydrogen bond with Lys 161. The flexible hydrophilic N,N-dimethyl ethyl amino moiety extended to the solvent front area, with the nitrogen atom in this moiety forming a hydrogen bond with the Ser169 residue (Fig. 2b). Therefore, even though osimertinib is an irreversible inhibitor of EGFR, our docking analysis showed that it binds to MNK2 in a reversible manner. Consistently, our washout experiment in primary GBM cell lysates (GBM-1) revealed that elF4E phosphorylation quickly recovered after the compound was removed, which further confirmed this reversible binding mode (Fig. 2c). Since elF4E plays an important role in mRNA translation, we employed the cap-binding assay to examine the effect of osimertinib on cap-dependent translation. elF4A and elF4G are members of the elF4G cap-binding complex. Treatment of primary GBM cells with osimertinib suppressed the activity of elF4E and reduced elF4A and elF4G to the $5^{\prime}$ cap (Fig. 2d). Next, we measured protein synthesis using the SUnSET assay and found that the cells treated with osimertinib incorporated less puromycin than control cells. These results showed that osimertinib reduces protein synthesis by affecting cap-dependent translation (Fig. 2e). Next, we compared the antiproliferative activities of osimertinib, tomivosertib (MNK inhibitor), and erlotinib (EGFR inhibitor) against three drug-sensitive primary GBM cell samples (Supplementary Table. S1). The results showed that osimertinib and tomivosertib could effectively suppress the growth of primary GBM cells, but erlotinib had no apparent effect (Fig. 2f). Furthermore, the colony-formation assay displayed similar results (Supplementary Fig. S1). These results suggested that osimertinib might target MNKs rather than EGFR to achieve its antiproliferative efficacy. We then investigated the effect of osimertinib on MNK signaling in drug-sensitive primary GBM cells with no detectable EGFR expression. Both osimertinib and the MNK inhibitor tomivosertib suppressed the phosphorylation of elF4E, a well-known downstream target of MNKs (Fig. 2g), but neither of them affected the phosphorylation of ERK, a downstream effector of EGFR. In addition, we found that elF4E phosphorylation was inhibited only at a high concentration of osimertinib $(10 \mu \mathrm{M})$ in osimertinib-nonresponsive GBM cells; however, it had no effect on cell proliferation (Supplementary Fig. S2). Collectively, these findings suggested that pharmacological blockade of MNKs may be beneficial for EGFR-negative GBM.

Osimertinib shows in vivo anticancer efficacy in patient-derived tumor xenograft (PDX) models

To test the in vivo antitumor efficacy of osimertinib, we examined tumor growth in GBM patient-derived xenograft models. Brain section staining showed that the tumors were visibly smaller in animals treated with osimertinib and temozolomide than the 
$\mathbf{a}$

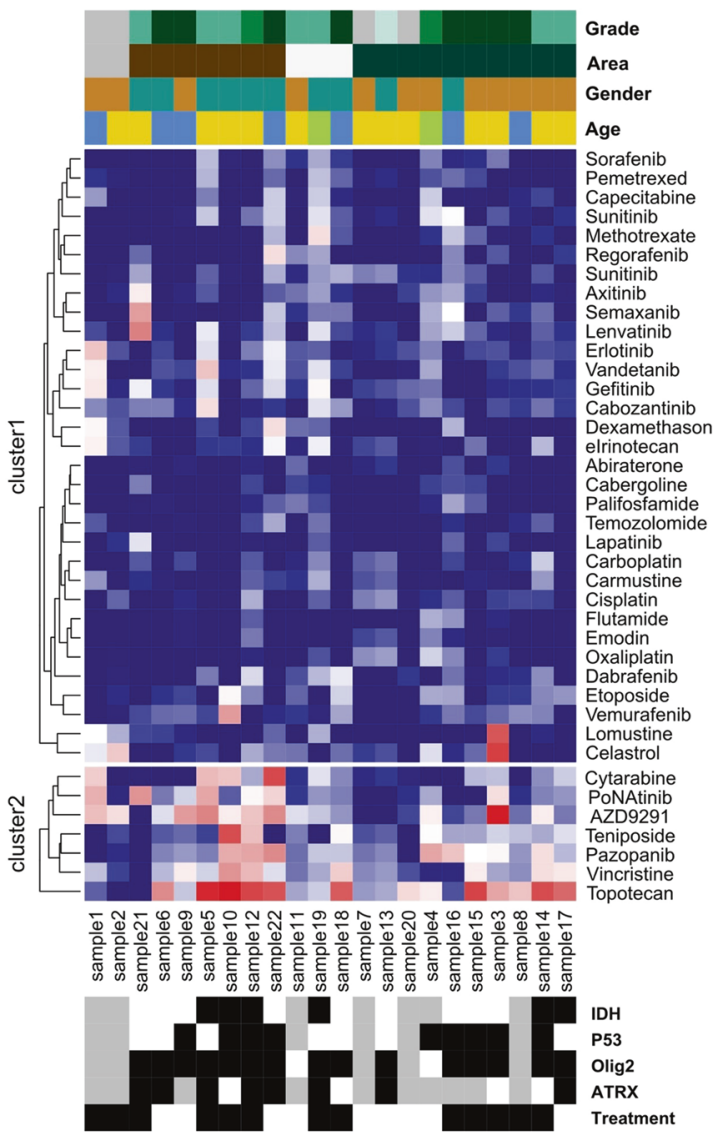

b
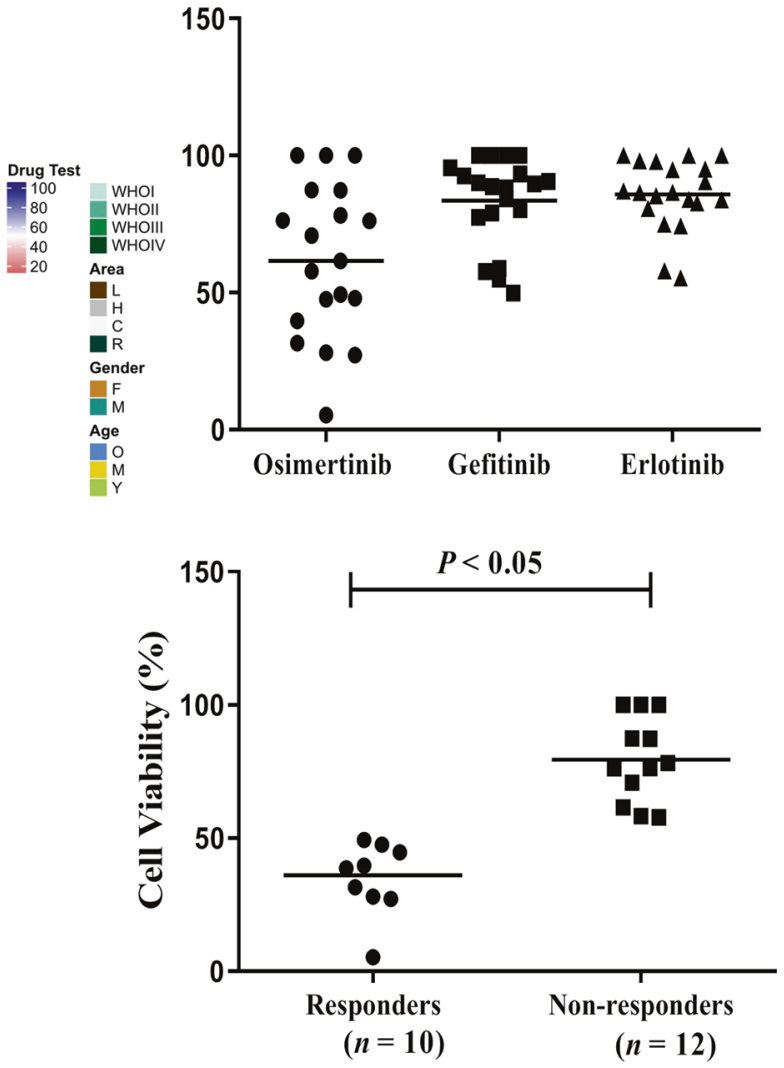

c

d

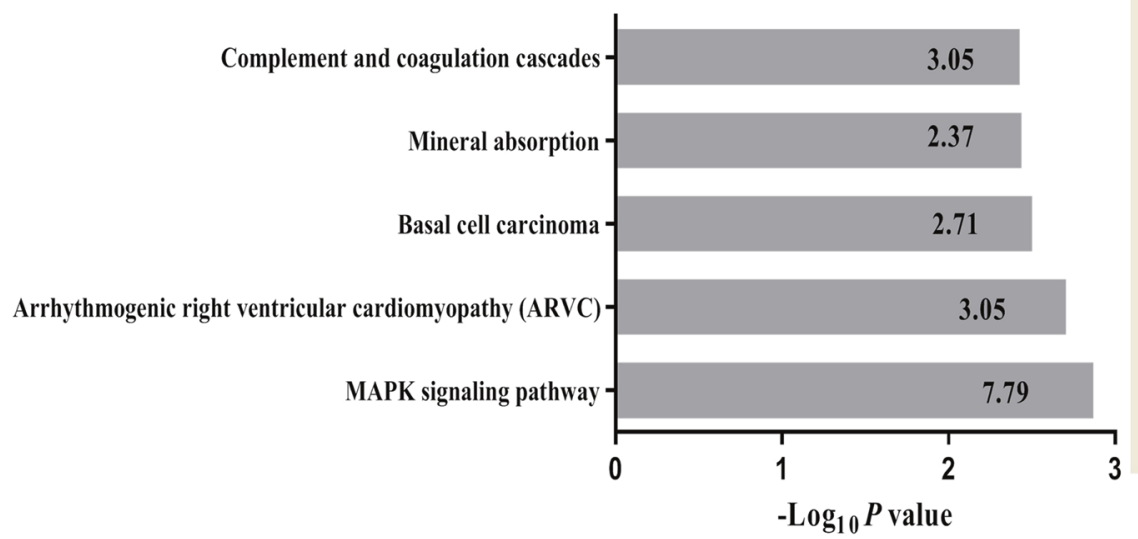

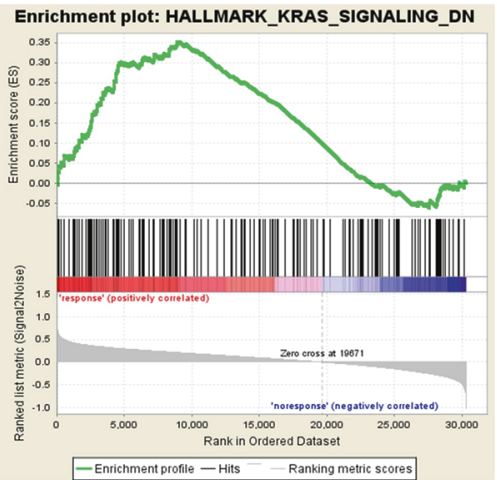

Fig. 1 Effects of osimertinib on patient-derived primary glioblastoma cells. a Analysis of drug sensitivity, pathological features, and patient characteristics. Drug sensitivity (red) and resistance (blue) are annotated by a color gradient, and the heatmap is annotated at the top and bottom with patient characteristics, disease grade, disease location, and pathological features as indicated in the key. b Drug sensitivity analysis of EGFR inhibitors. c Kyoto Encyclopedia of Genes and Genomes (KEGG) pathway enrichment analysis for upregulated and downregulated genes between drug-sensitive and drug-insensitive primary GBM cells. d GSEA of RNA-sequencing data for the patientderived primary glioblastoma cells.

vehicle group (Fig. 3a). Oral administration of osimertinib at a $40 \mathrm{mg} \cdot \mathrm{kg}^{-1} \cdot \mathrm{d}^{-1}$ dosage almost completely suppressed the tumor progression in five out of six animals (average tumor growth inhibition $(\mathrm{TGl})=74.5 \%$, with no apparent toxicity observed during the treatment. Temozolomide at $40 \mathrm{mg} \cdot \mathrm{kg}^{-1} \cdot \mathrm{d}^{-1}$ dosage also showed significant tumor growth reduction (average $\mathrm{TGI}=$ 54.7\%) (Fig. 3b). In addition, immunohistochemistry staining and Western blot demonstrated that elF4E phosphorylation was greatly inhibited in tumor cells upon osimertinib treatment (Fig. 3c, d). The results of immunohistochemistry and TUNEL assays showed that osimertinib $(40 \mathrm{mg} / \mathrm{kg})$ potently decreased the proliferation of Ki67-positive tumor cells and led to a clear increase in the apoptosis of TUNEL-positive cells compared to the vehicle control (Fig. 3d). Taken together, these results demonstrated that osimertinib targets MNKs in GBM tumors and shows promising tumorsuppressing activity in PDX models. 
$\mathbf{a}$

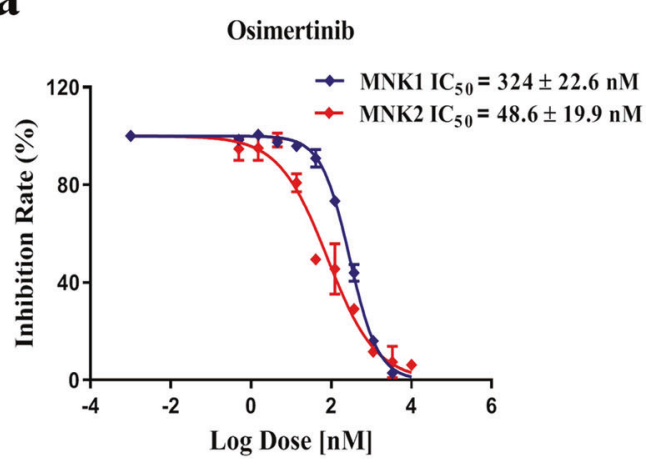

c

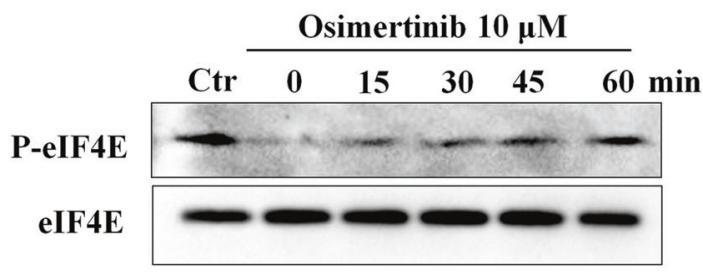

b
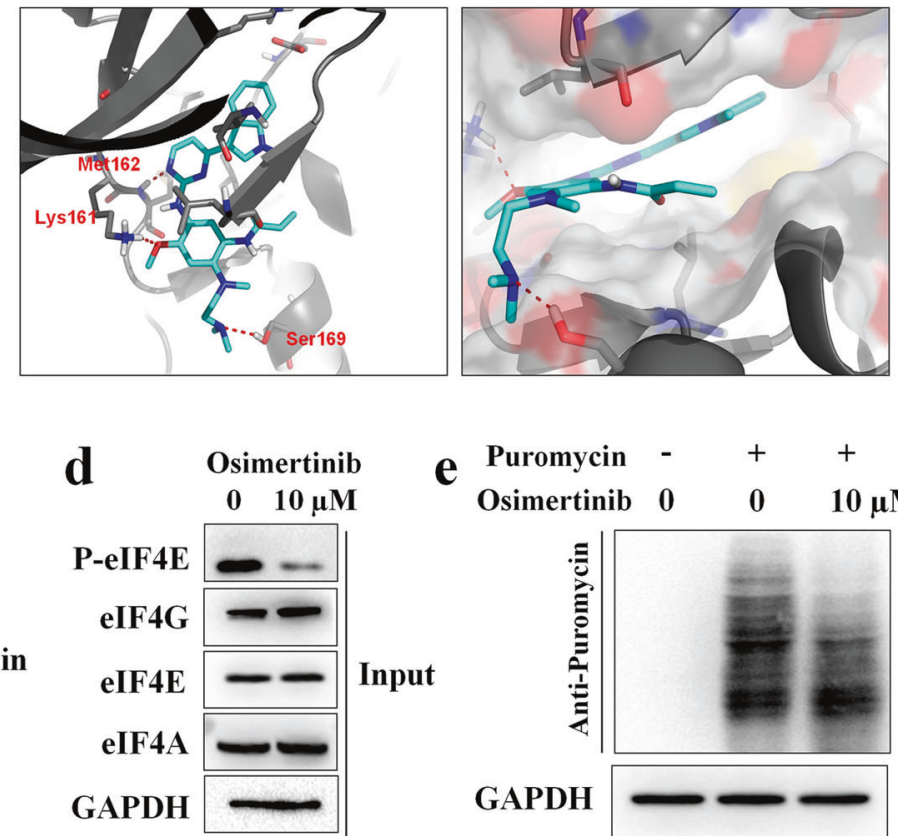

$\begin{array}{cccc}\text { Puromycin } & - & + & + \\ \text { Osimertinib } & 0 & 0 & 10 \mu \mathrm{M}\end{array}$

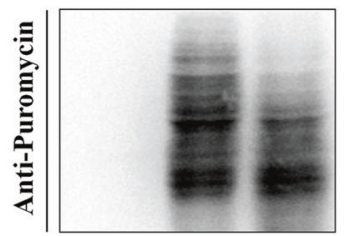

GAPDH

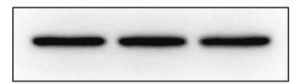

$m^{7}$ GTP pull-down Osimertinib
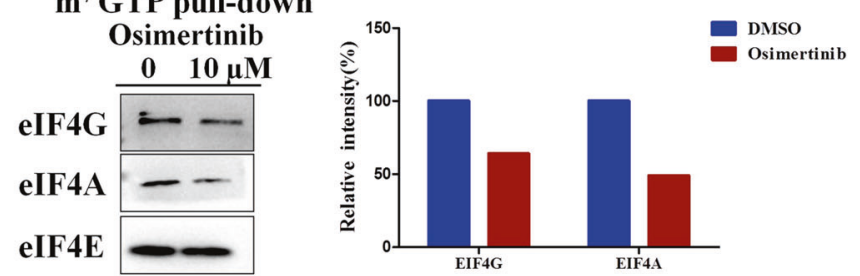

f

GBM-1

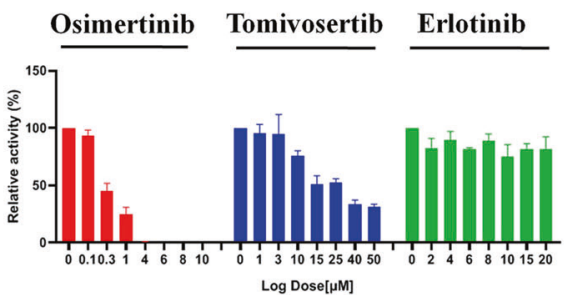

g

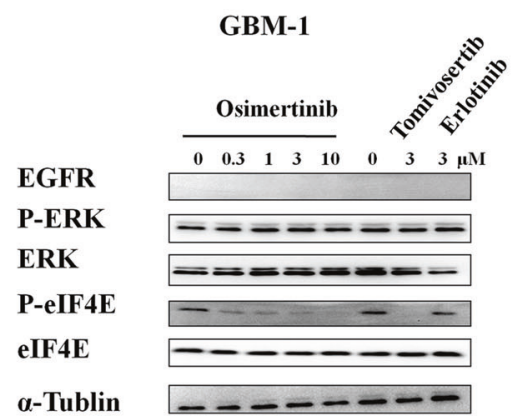

GBM-2

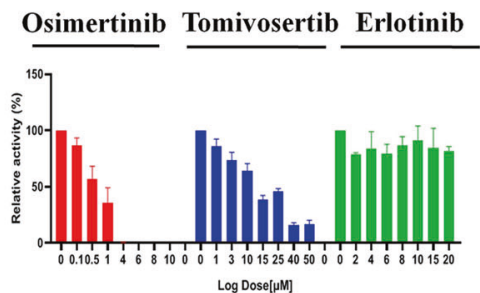

GBM-2

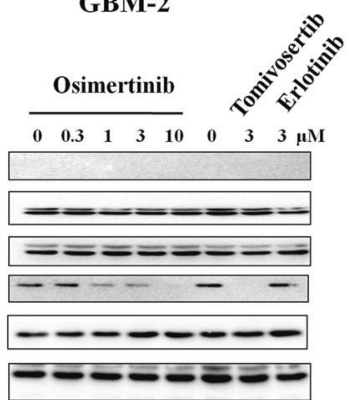

GBM-3

Osimertinib Tomivosertib Erlotinib

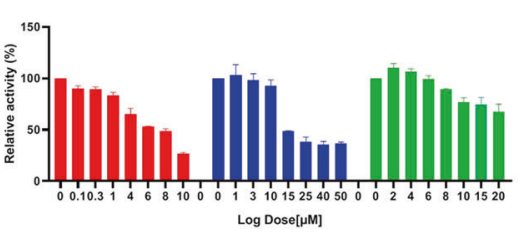

GBM-3

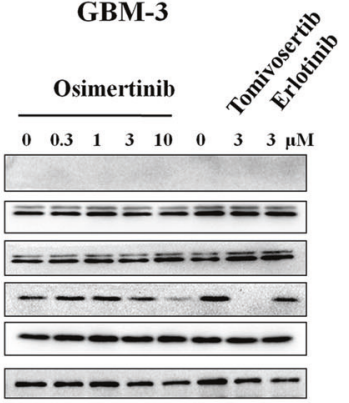

Fig. 2 Characterization of osimertinib as a highly potent MNK inhibitor in GBM cells. a Invitrogen Z'LYTE biochemical assay determination of the IC ${ }_{50}$ values of osimertinib against MNKs. $\mathbf{b}$ Molecular docking of MNK2 (PDB ID: 6CJH) in complex with osimertinib. c Inhibitory effect of osimertinib on MNK signaling in primary GBM cells at different time points after $4 \mathrm{~h}$ of pretreatment and removal of the drug. $\mathbf{d}$ Primary GBM cells were treated with $10 \mu \mathrm{M}$ osimertinib for $6 \mathrm{~h}$. Whole-cell extracts were incubated with $\mathrm{m}^{7} \mathrm{GTP}$-agarose beads overnight. The $\mathrm{m}^{7} \mathrm{GTP}$ agarose input control and pull-down were subjected to SDS-PAGE followed by immunoblotting with antibodies against phospho-elF4E, elF4G, elF4E, elF4A, and GAPDH. e Protein synthesis in primary cells was assessed using a puromycin incorporation assay with immunoblotting. f Antitumor activities of osimertinib in EGFR-negative primary GBM cells. $g$ Effects of osimertinib on EGFR- and MNK-mediated signaling pathways. 


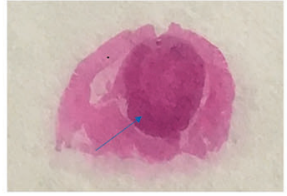

Vehicle

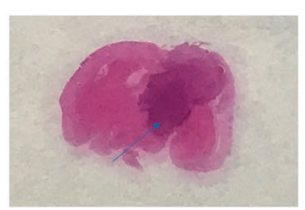

Osimertinib $20 \mathrm{mg} / \mathrm{kg}$

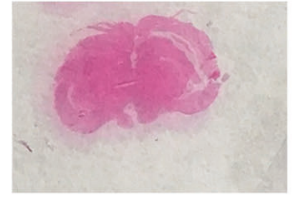

Osimertinib $40 \mathrm{mg} / \mathrm{kg}$

b
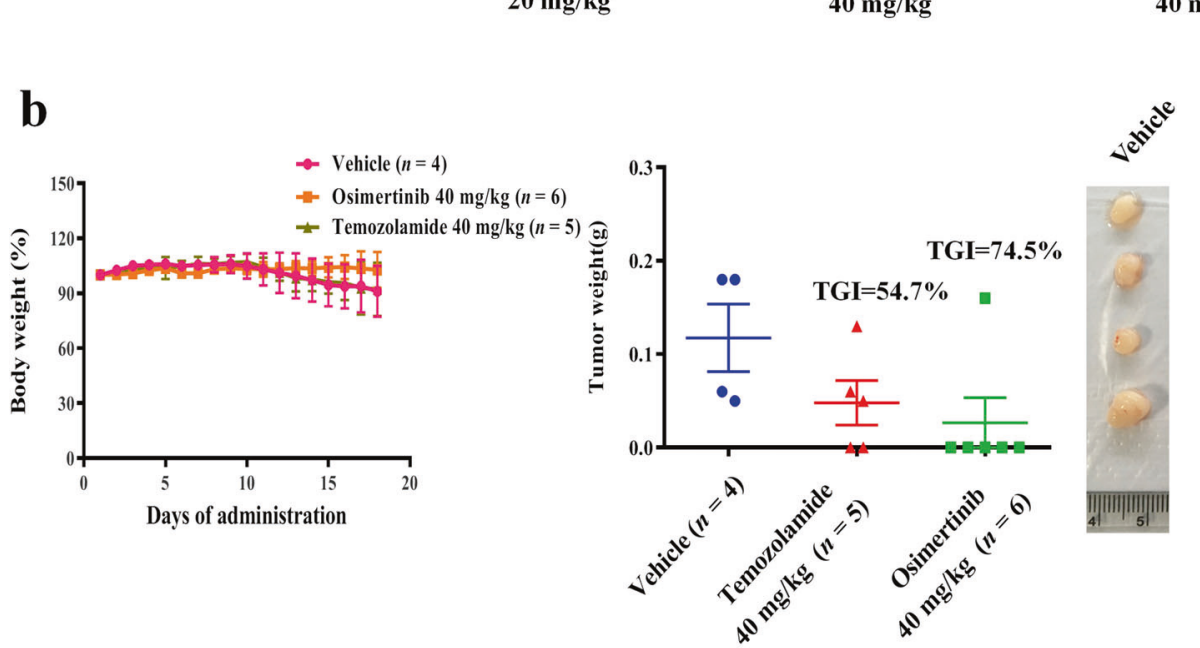

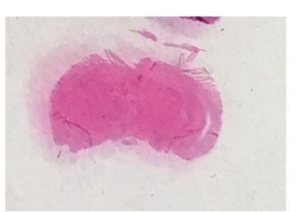

Temozolamide $40 \mathrm{mg} / \mathrm{kg}$

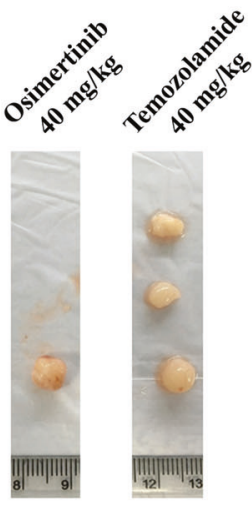

c

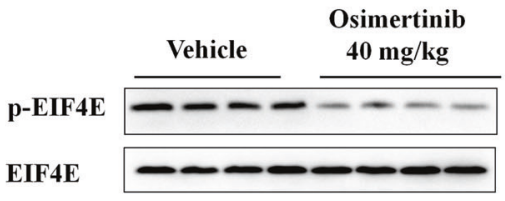

d

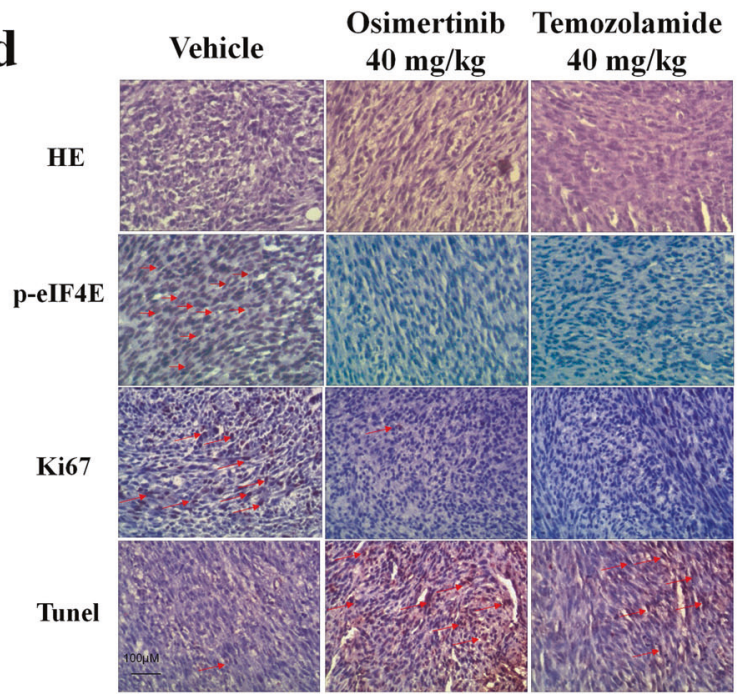

Fig. 3 Antitumor efficacy of osimertinib in GBM patient-derived xenograft mouse models. a Representative pictures of brain sections from the vehicle, osimertinib and temozolomide treatment groups with H\&E staining. $\mathbf{b}$ Osimertinib slowed tumor progression in PDX models at the $40 \mathrm{mg} / \mathrm{kg}$ qd dosage. c Osimertinib inhibited MNK activity in vivo according to Western blot analysis. d Representative pictures of immunohistochemical staining of brain tumors from vehicle- and osimertinib-treated mice. H\&E staining (top), IHC for phospho-elF4E (Ser209), Ki-67, and TUNEL analyses are shown.

\section{DISCUSSION}

Despite improved early diagnosis and combination therapy, GBM patients still have a poor prognosis and high tumor recurrence rates. Generally, drug repurposing is much more time and cost efficient than traditional drug discovery routes for seeking new available therapies. In this study, in a cohort of tumor samples from GBM patients, we analyzed transcriptomic data and in vitro drug sensitivity and discovered that osimertinib was effective against a subset of EGFR-negative GBM samples. Osimertinib, a thirdgeneration EGFR inhibitor, was approved for the treatment of non-small-cell lung cancer patients with the EGFR T790M mutation and brain metastasis [13, 14]. Notably, osimertinib has superior properties for penetrating the BBB, and recent studies showed that osimertinib displayed antitumor efficacy in GBM patients with constitutively active EGFR due to abnormal splicing [15]. Interestingly, our results showed that osimertinib was still effective in the absence of activated EGFR primary GBM cells. To determine how osimertinib achieved the antitumor properties, we performed RNAseq assays and found that the MAPK pathway might mediate the effects of osimertinib against EGFR-negative GBM cells.

As the downstream effectors of MAPK-signaling, MNKs control protein synthesis by regulating the activity of elF4E and thus play an important role in development of GBM [16-18]. We found that the MAPK pathway is upregulated in a subset of GBM patient cells, and osimertinib noncovalently binds to MNK and displays potent biochemical inhibition of MNKs $[19,20]$. Osimertinib showed 
significant antiproliferative effects against primary GBM cells as well as in vivo tumor suppression in PDX models. However, according to the kinase profiling results, osimertinib is a multitarget kinase inhibitor that can also inhibit ALK, FGFR1, FAK, etc [21-26]. These targets are related to the occurrence and development of GBM. Therefore, osimertinib may inhibit multiple kinase targets.

Taken together, our results have shown that osimertinib effectively inhibits the growth of EGFR-negative GBM cells by targeting MNKs. Given that osimertinib has been clinically approved with an acceptable safety profile and good BBB permeability, our results might help to expand the application of osimertinib to EGFR-negative GBM patients.

\section{ACKNOWLEDGEMENTS}

This study was supported by the National Natural Science Foundation of China (grants 81773777, 81673469, 81703559, and 81172407), the Natural Science Foundation of Anhui Province (grants 1808085MH274 and 1908085MH259), the China Post-doctoral Science Foundation (grants 2018T110634, 2018M630720, and 2019M652057), the Post-doctoral Science Foundation of Anhui Province (grant 2018B279), Science and Technology Project grants from Anhui Province (grants 1508085QHI84, 1606c08235, and 1604a0802069), the Fundamental Research Fund for Central Universities (WK 9110000032), the Frontier Science Key Research Program of the Chinese Academy of Sciences (grant QYZDB-SSW-SLH037), the CASHIPS Director's Fund (grant BJPY2019A03), the Key Program of the 13th Five-Year Plan of CASHIPS (grant KP-2017-26), and the Presidential Foundation of CASHIPS (grant YZJJ2018QN17). A portion of this work was supported by the High Magnetic Field Laboratory of Anhui Province.

\section{AUTHOR CONTRIBUTIONS}

QSL, CSN, and WCW designed the project and wrote the manuscript. CC, CDC, HW, LW, ZWW, and ZRJ performed the experiments and collected the data. CC, HW, ALW, and $\mathrm{CH}$ drafted the manuscript. YFD, WXN, and SQ analyzed the data. ZPQ and JL revised the manuscript. All the authors read and approved the final manuscript.

\section{ADDITIONAL INFORMATION}

The online version of this article (https://doi.org/10.1038/s41401-020-0418-2) contains supplementary material, which is available to authorized users.

Competing interests: The authors declare no competing interests.

\section{REFERENCES}

1. Nizamutdinov D, Stock EM, Dandashi JA, Vasquez EA, Mao Y, Dayawansa S, et al. Prognostication of survival outcomes in patients diagnosed with glioblastoma. World Neurosurg. 2018;109:e67-e74.

2. Hanif F, Muzaffar K, Perveen K, Malhi SM, Simjee ShU. Glioblastoma multiforme: a review of its epidemiology and pathogenesis through clinical presentation and treatment. Asian Pac J Cancer Prev. 2017;18:3-9.

3. de Robles P, Fiest KM, Frolkis AD, Pringsheim T, Atta C, St Germaine-Smith C, et al. The worldwide incidence and prevalence of primary brain tumors: a systematic review and meta-analysis. Neuro Oncol. 2015;17:776-83.

4. Bell JB, Eckerdt FD, Alley K, Magnusson LP, Hussain H, Bi Y, et al. MNK inhibition disrupts mesenchymal glioma stem cells and prolongs survival in a mouse model of glioblastoma. Mol Cancer Res. 2016;14:984-93.
5. Fukunaga R, Hunter T. MNK1, a new MAP kinase-activated protein kinase, isolated by a novel expression screening method for identifying protein kinase substrates. EMBO J. 1997;16:1921-33.

6. Proud CG. Mnks, elF4E phosphorylation and cancer. Biochim Biophys Acta. 2015;1849:766-73.

7. Graff JR, Konicek BW, Carter JH, Marcusson EG. Targeting the eukaryotic translation initiation factor $4 \mathrm{E}$ for cancer therapy. Cancer Res. 2008;68:631-4.

8. Ruggero D, Montanaro L, Ma L, Xu W, Londei P, Cordon-Cardo C, et al. The translation factor elF-4E promotes tumor formation and cooperates with c-Myc in lymphomagenesis. Nat Med. 2004;10:484-6.

9. Hou J, Lam F, Proud C, Wang S. Targeting mnks for cancer therapy. Oncotarget. 2012;3:118-31.

10. Diab S, Kumarasiri M, Yu M, Teo T, Proud C, Milne R, et al. MAP kinase-interacting kinases-emerging targets against cancer. Chem Biol. 2014;21:441-52.

11. Truitt ML, Conn CS, Shi Z, Pang X, Tokuyasu T, Coady AM, et al. Differential requirements for elF4E dose in normal development and cancer. Cell. 2015;162:59-71.

12. Schmidt EK, Clavarino G, Ceppi M, Pierre P. SUnSET, a nonradioactive method to monitor protein synthesis. Nat Methods. 2009;6:275-7.

13. Cross DA, Ashton SE, Ghiorghiu S, Eberlein C, Nebhan CA, Spitzler PJ, et al. AZD9291, an irreversible EGFR TKI, overcomes T790M-mediated resistance to EGFR inhibitors in lung cancer. Cancer Discov. 2014;4:1046-61.

14. Lee SY. Temozolomide resistance in glioblastoma multiforme. Gene Dis. 2016:3:198-210.

15. Colclough N, Ballard PG, Barton P, Chen K, Cross DAE, Finlay MRV, et al. Preclinical comparison of the blood brain barrier (BBB) permeability of osimertinib (AZD9291) with other irreversible next generation EGFR TKIs. Eur J Cancer. 2016;69:S28

16. Ballard P, Yates JW, Yang Z, Kim DW, Yang JC, Cantarini M, et al. Preclinical comparison of osimertinib with other EGFR-TKIs in EGFR-mutant NSCLC brain metastases models, and early evidence of clinical brain metastases activity. Clin Cancer Res. 2016;22:5130-40.

17. Liu X, Chen X, Shi L, Shan Q, Cao Q, Yue C, et al. The third-generation EGFR inhibitor AZD9291 overcomes primary resistance by continuously blocking ERK signaling in glioblastoma. J Exp Clin Cancer Res. 2019;38:219.

18. Grzmil M, Seebacher J, Hess D, Behe M, Schibli R, Moncayo G, et al. Inhibition of MNK pathways enhances cancer cell response to chemotherapy with temozolomide and targeted radionuclide therapy. Cell Signal. 2016;28:1412-21.

19. Grzmil M, Morin P Jr, Lino MM, Merlo A, Frank S, Wang Y, et al. MAP kinaseinteracting kinase 1 regulates SMAD2-dependent TGF-beta signaling pathway in human glioblastoma. Cancer Res. 2011;71:2392-402.

20. Bell JB, Eckerdt FD, Alley K, Magnusson LP, Hussain H, Bi Y, et al. MNK inhibition disrupts mesenchymal glioma stem cells and prolongs survival in a mouse model of glioblastoma. Mol Cancer Res. 2016;14:984-93.

21. Finlay MR, Anderton $M$, Ashton $S$, Ballard $P$, Bethel $P A, B o x M R$, et al. Discovery of a potent and selective EGFR inhibitor (AZD9291) of both sensitizing and T790M resistance mutations that spares the wild type form of the receptor. J Med Chem. 2014;57:8249-67.

22. Gilani A, Donson A, Davies KD, Whiteway SL, Lake J, DeSisto J, et al. Targetable molecular alterations in congenital glioblastoma. J Neurooncol. 2020;146:247-52.

23. Chiba R, Akiya $M$, Hashimura $M$, Oguri $Y$, Inukai $M$, Hara $A$, et al. ALK signaling cascade confers multiple advantages to glioblastoma cells through neovascularization and cell proliferation. PLoS ONE. 2017;12:e0183516.

24. Junca A, Villalva C, Tachon G, Rivet $P$, Cortes U, Guilloteau K, et al. Crizotinib targets in glioblastoma stem cells. Cancer Med. 2017;6:2625-34.

25. Jimenez-Pascual A, Siebzehnrubl FA. Fibroblast growth factor receptor functions in glioblastoma. Cells. 2019;8:E715.

26. Natarajan M, Hecker TP, Gladson CL. FAK signaling in anaplastic astrocytoma and glioblastoma tumors. Cancer J. 2003;9:126-33. 